\title{
$\operatorname{arCOS} D E S I G N$
}

\section{A utilização de modelos 3D para a preservação e divulgação de peças do patrimônio histórico e cultural: estudo de caso com escarradeira e urinol}

Wagner Soares Rossi (UFRGS, Brasil)

wsrossi@gmail.com

Avenida Paulo Gama, 110, Bairro Farroupilha

Porto Alegre, Rio Grande do Sul, Brasil CEP: 90040-060

Fábio Pinto da Silva (UFRGS, Brasil)

fabio.silva@ufrgs.br

Wilson Kindlein Júnior (UFRGS, Brasil)

kindlein@portoweb.com.br 


\title{
A utilização de modelos 3D para a preservação e divulga- ção de peças do patrimônio histórico e cultural: estudo de caso com escarradeira e urinol
}

Resumo: As Tecnologias da Informação e Comunicação por meio da disponibilização de modelos tridimensionais podem contribuir de maneira significativa para a divulgação e aproximação do acervo dos museus com a comunidade. Dessa maneira, este artigo apresenta a utilização da técnica de digitalização tridimensional para a construção de modelos 3D de duas peças de acervo de museu: a escarradeira e o urinol. A digitalização foi feita com um Scanner 3D que utiliza um sensor conoscópico a Laser, instalado no Laboratório de Design e Seleção de Materiais da Universidade Federal do Rio Grande do Sul. Os dados foram processados em softwares de modelagem 3D e 2D para a obtenção dos modelos virtuais. Ao final do processo, foram obtidos modelos 3D fidedignos às peças originais, o que sinaliza a possibilidade de sua utilização para a representação virtual das peças contribuindo assim para preservação e divulgação de peças do patrimônio histórico cultural.

Palavras-chave: digitalização 3D, museu, patrimônio histórico.

\section{The use of 3D models for the preservation and dissemination of pieces of historical and cultural}

\begin{abstract}
Information Technologies and Communication through the availability of 3D models can contribute to the dissemination and approximate of museums with the community. Thus, this paper presents the use of three-dimensional scanning technique to construct 3D models of two pieces of museum's collection: a spittoon and urinal. The scan was made with a 3D scanner that uses a sensor conoscopic Laser, at the Laboratory of Design and Materials Selection of the Federal University of Rio Grande do Sul. The data were processed in software 3D modeling and 2D models to obtain the virtual models. At the end of the process, 3D models reliable to were obtained the original parts, which indicates the possibility of their use for the virtual representation of the parts contributing to the preservation and dissemination of cultural heritage pieces.
\end{abstract}

Key words: 3D scanning, museum, heritage. 


\section{Introdução}

A expansão das Tecnologias da Informação e Comunicação pode contribuir de maneira significativa para a aproximação do acervo dos museus com a comunidade, facilitando o acesso às coleções independentemente da localização e também do tempo, já que as coleções virtuais se encontram mais acessíveis. Uma das maneiras de tornar essa coleção acessível virtualmente é por meio da utilização das técnicas de digitalização 3D em peças disponíveis fisicamente nos museus, contribuindo, assim, para a divulgação e preservação da memória do patrimônio. Em museologia as ferramentas de uso geral para a digitalização 3D e renderização provaram ser extremamente úteis no apoio à visualização interativa (ARBACE et al., 2013) e na construção de coleções de referência virtual (BETTS et al., 2011).

A tecnologia de reprodução de modelos 3D digitais têm sido muito utilizada para atividades de conservação ou restauração. Arbace et al. (2013) cita algumas experiências representativas na área: execução de investigações com o apoio de documentação digital; reconstrução virtual de obras de arte fragmentadas; projeto de estruturas de proteção para garantir transporte seguro de artefatos delicados; uso de modelos digitalizados como suporte para o planejamento de restauração por meio do cálculo centro de gravidade e peso para objetos pesados; produção de cópias com as tecnologias digitais de fabricação e metodologias que permitam simular e prever os efeitos do envelhecimento sobre os materiais.

Embora a principal motivação para desenvolver modelos 3D a partir de peças de museu seja para a documentação, conservação e análise, existe também um interesse pela utilização para a visualização interativa (GODIN et al., 2002 e BETTS et al., 2011). Os modelos 3D podem ser manipulados em tempo real, girados e ampliados em qualquer posição e ponto de vista, fornecendo dessa maneira diferentes possibilidades de aprendizagem tornando as peças acessíveis on-line. Além da aprendizagem, os modelos 3D disponíveis na web oferecem mais três benefícios críticos quando comparados às maneiras tradicionais de visualização de coleções de museus, como no caso dos guias ilustrados em papel: resolução e detalhes superiores; facilidade de atualização e correção; acesso fácil e gratuito pela Internet (вETTs et al., 2011).

O uso da tecnologia da digitalização 3D em museus se apresenta como uma alternativa interessante devido às diversas possibilidades de utilização, podendo-se aplicar em diferentes coleções de um acervo. Segundo Silva et al. (2010) a digitalização 3D apresenta a possibilidade de digitalizar uma infinidade de objetos, que vão desde peças de joalheria até mesmo partes do corpo humano. 
Procurando explorar as possibilidades de utilização da digitalização 3D para a construção de modelos virtuais para a divulgação e preservação da memória e do patrimônio, este trabalho apresenta a utilização da técnica de digitalização 3D para produzir modelos virtuais de duas peças do Museu da cidade de Porto Alegre Joaquim José Felizardo: a escarradeira e o urinol.

\section{Museu Joaquim José Felizardo}

O Museu Histórico da cidade de Porto Alegre, localizado no estado do Rio Grande do Sul (Rs), denominado Joaquim José Felizardo, foi criado pelo decreto 6.598 de 13 de março de 1979, como Museu Municipal e está situado em um prédio tombado pelo Patrimônio Histórico Municipal de Porto Alegre em. Como disse um de seus primeiros diretores: "o próprio prédio é parte do acervo do museu” (PREFEITURA MUNICIPAL DE PORTO ALEGRE, 2013).

O prédio, localizado na Rua João Alfredo, 582, no bairro Cidade Baixa, em uma casa conhecida como o Solar Lopo Gonçalves, foi construído entre 1845 e 1855, na antiga rua da Margem. Com arquitetura de influência luso-brasileira, foi concebido para ser "residência da chácara", lugar de descanso da família do comerciante português Lopo Gonçalves, nos fins de semana e feriados.

O Museu conta com três importantes acervos sobre a História de Porto Alegre - RS: o acervo histórico, o acervo fotográfico e o acervo arqueológico. O acervo histórico é composto por cerca de 1.300 peças, do final do século XIx e do século xx. O acervo fotográfico é composto por aproximadamente 20.000 fotografias e o arqueológico compreende 120.000 peças, entre fragmentos e objetos inteiros. Tratam-se de peças cerâmicas, líticas, peças em louça, em vidro, em metal, em couro e em osso.

As obras selecionadas para a digitalização 3D fazem parte do acervo arqueológico e foram escolhidas pela equipe do próprio museu por se tratarem de duas das peças mais importantes do acervo: a escarradeira e o urinol. As informações sobre as peças foram fornecidas por Tocchetto (2013) e apresentadas a seguir.

\subsection{A escarradeira}

A Escarradeira ou cuspideira (Figura 1) em porcelana é uma peça de origem europeia que data do final do século XIX, decorada com a técnica de pintura a mão livre. Era utilizada para expectorar pigarro, saliva e mucos produzidos por doenças da época, como a tuberculose. 

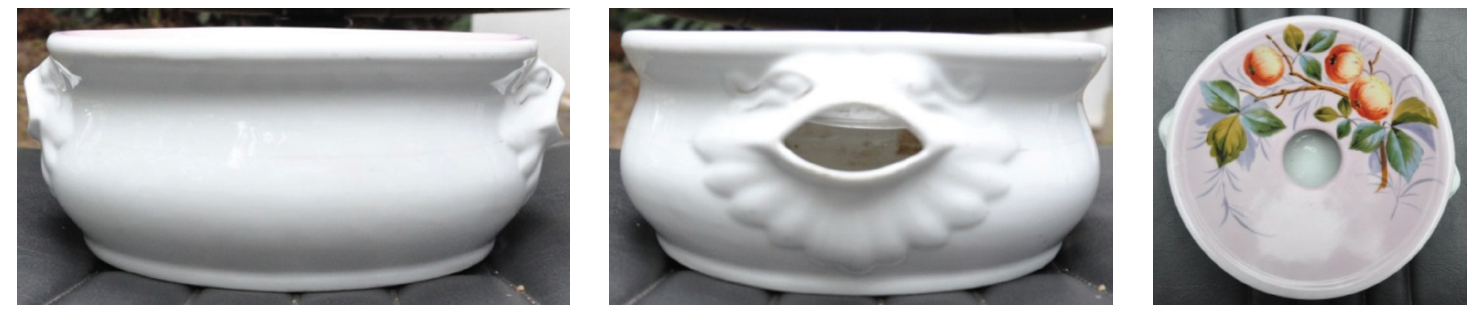

Figura 1. Escarradeira do acervo do museu de Porto Alegre - RS.

A peça era colocada no chão, ladeando os sofás das salas e nos gabinetes de fumantes das casas. Sua importância se dá, pois, ela compunha o mobiliário das residências urbanas no século XIx. Era considerada um bem supérfluo, adquirido somente pelas famílias abastadas. O Museu de Porto Alegre recebeu a peça por doação de seus proprietários João Manoel de Castro e Edi Aleda Rosa, moradores de Porto Alegre.

\subsection{O urinol}

O Urinol (Figura 2) em faiança (cerâmica de baixa temperatura) fina europeia foi produzido entre os anos 1830 e 1860, decorado também com a técnica de pintura a mão livre e carimbado. O uso de urinóis no recolhimento de matérias fecais e urina tinha seu uso comum, principalmente, antes da disseminação dos vasos sanitários.
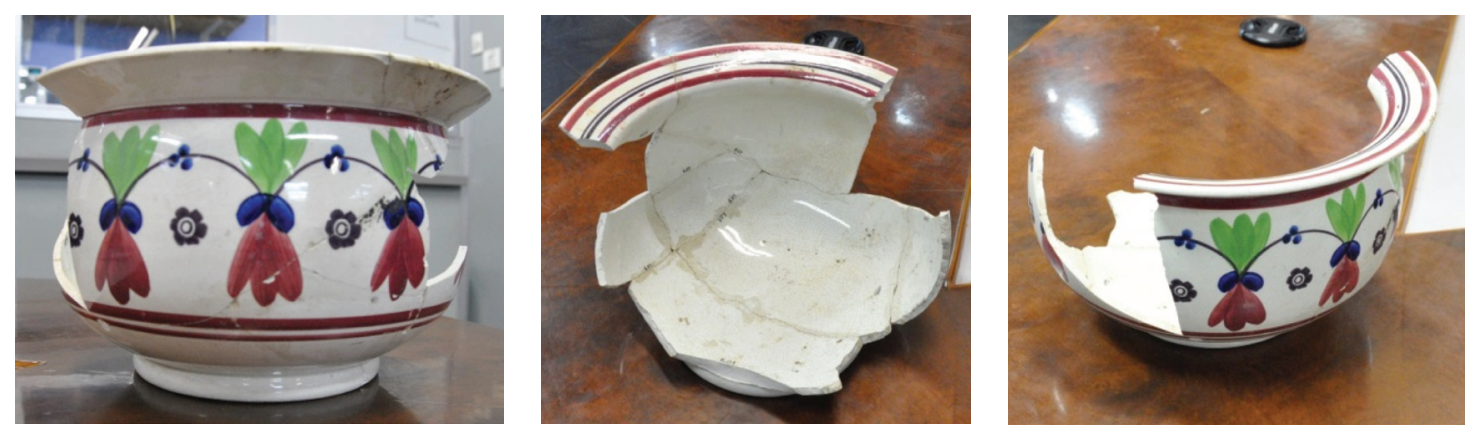

Figura 2. Urinol do acervo do museu de Porto Alegre - RS.

Trata-se de uma peça arqueológica, coletada no sítio arqueológico Praça Rui Barbosa, localizado onde hoje se encontra o Centro Popular de Compras. A peça foi recuperada em 1995 por ocasião das reformas no terminal de ônibus que lá existia. A área, no século XIx, era ocupada por estaleiros e oficinas, além de ser um depósito de lixo urbano. 


\section{Materiais e Métodos}

A digitalização tridimensional é uma técnica utilizada para capturar imagens e dados de objetos em 3D utilizando-se do auxílio de ferramentas computacionais é possível à captura de imagens em alta resolução e alta precisão de informações não só sobre a forma, mas também sobre detalhes da superfície e variações, permitindo também obter com grande precisão curvas, detalhes de superfícies e texturas (вORGEAT et al., 2007 e sILVA, 2011). A digitalização 3D também permite que se realizem medidas de desgaste, construção de moldes, análise de superfícies, para isso, normalmente, são utilizados sistemas de alta tecnologia, como o Scanner 3D e softwares CAD/CAE/CAM (sILVA, 2011).

A grande variedade de formas, tamanhos e materiais, que caracterizam o domínio de obras de um museu tem impacto significativo na escolha da tecnologia mais adequada para a digitalização. Esta é muito influenciada pelas características físicas do objeto a ser digitalizado, incluindo o tamanho, a complexidade de sua superfície externa, as propriedades de reflexão da luz da superfície do objeto e até mesmo os possíveis impedimentos no acesso/ manipulação (ARBACE et al., 2013).

A utilização da técnica 3D para construir modelos virtuais a partir de modelos físicos é conhecida como Engenharia Reversa, pois ocorre em um processo inverso à engenharia convencional. Para isso são gerados dados de saída processados geometricamente como coordenadas da superfície no espaço tridimensional $(\mathrm{x}, \mathrm{y}, \mathrm{z})$. A varredura da superfície pode retornar milhares de pontos, chamados de 'nuvem de pontos'. O número de pontos está diretamente relacionado ao tamanho da área analisada e ao espaçamento entre pontos capturados (resolução). Esta nuvem de pontos, após manipulada computacionalmente, permite a geração de curvas, malhas, superfícies e sólidos tridimensionais que são compatíveis com sistemas CAD/CAE/CAM (sILVA, 2006).

As duas peças do museu, passaram pelo mesmo processo de digitalização, no Scanner 3D marca Tecnodrill, modelo Digimill 3D, que utiliza um sensor conoscópico a Laser. O equipamento está instalado no Laboratório de Design e Seleção de Materiais (LdsM) da Universidade Federal do Rio Grande do Sul (UFRGS). A Figura 3 apresenta o urinol e o equipamento utilizado. 


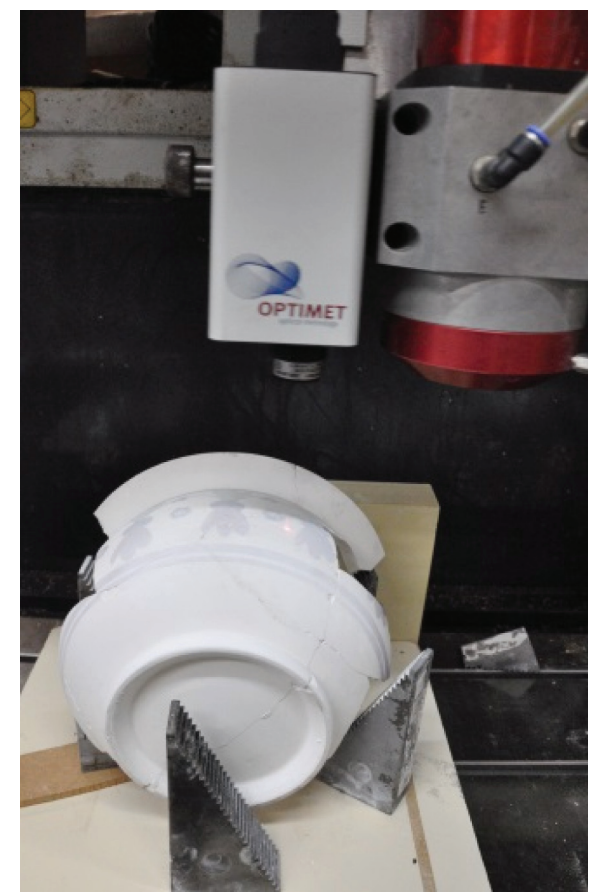

Figura 3. Urinol digitalizado no Scanner Tridimensional a Laser.

A primeira fase é a de preparação do equipamento e da amostra. A preparação do equipamento é feita por meio da seleção da lente e ajuste do eixo de varredura.

Foram selecionadas as lentes de acordo com o objeto: para a escarradeira foi utilizada uma lente de $100 \mathrm{~mm}$ e para o urinol uma de $250 \mathrm{~mm}$. A primeira possui uma faixa de captura (eixo z) de $35 \mathrm{~mm}$ com precisão de até $0,015 \mathrm{~mm}$, enquanto que a segunda captura $180 \mathrm{~mm}$ com precisão de $0,1 \mathrm{~mm}$. A amostra foi posicionada no scanner e preparada com um pó especial para eliminar os reflexos ocasionados pelo material reflexivo da peça. Após, foi feito o ajuste da qualidade, utilizando uma resolução em ambos os objetos de $0.2 \mathrm{~mm}$. Selecionou-se a área ( $\mathrm{x}, \mathrm{y}$ ) a ser digitalizada de acordo com o tamanho da peça. Os dados obtidos pela técnica de digitalização 3D foram editados em softwares de modelagem 3D (Geomagic Studio, 3D Studio Max), enquanto que a textura (cor) foi editada em software de edição de imagem 2D (Photoshop).

\section{Resultados e Discussão}

As nuvens de pontos receberam tratamentos de filtragem para remoção de ruídos e pontos indesejáveis, suavização, remoção de pontos sobrepostos entre diferentes nuvens, gerando uma malha de triângulos, exportada em formato STL, conforme Figura 4, a escarradeira e Figura 5, o urinol. 

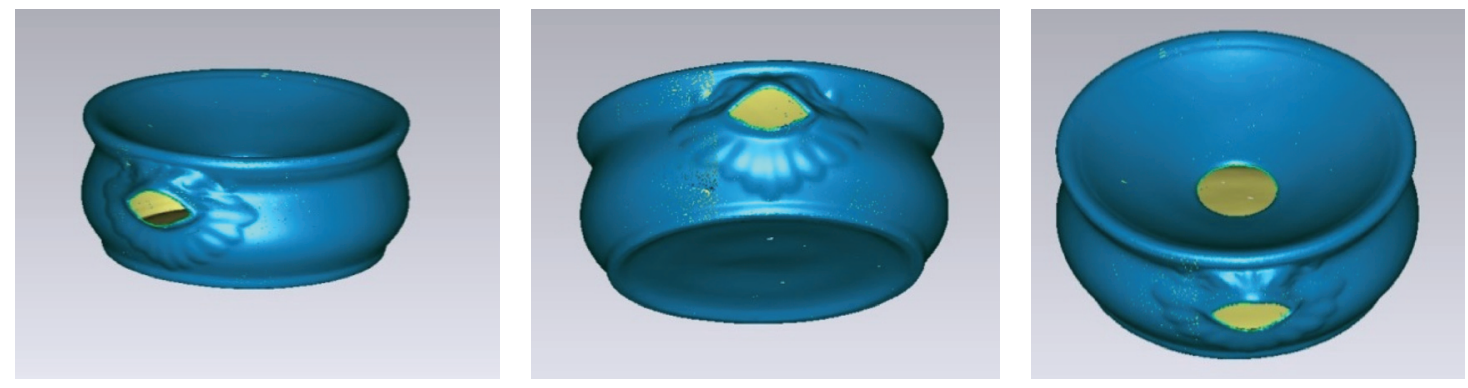

Figura 4. Malha de triângulos da escarradeira
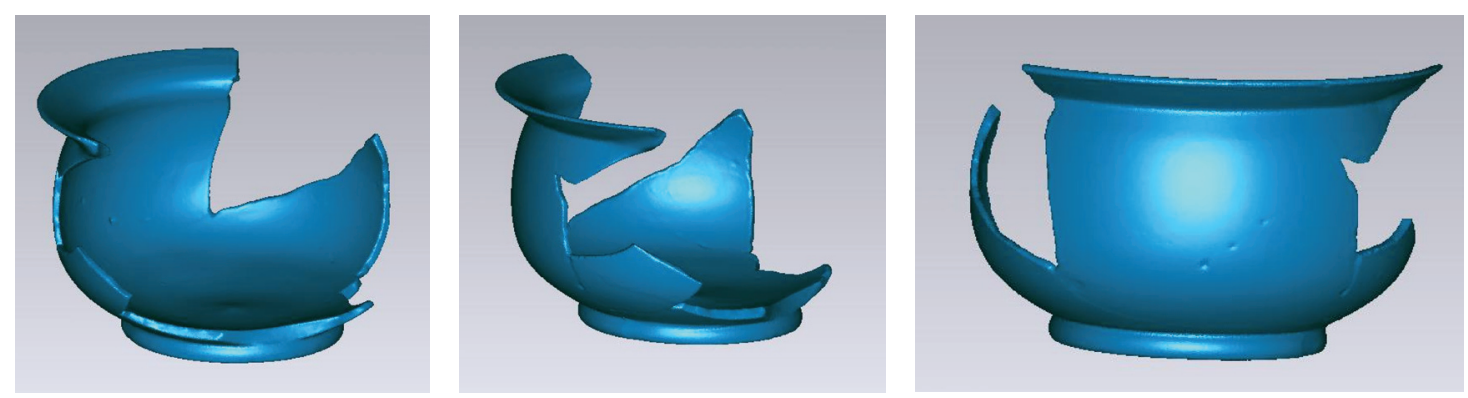

Figura 5. Malha de triângulos do urinol

O resultado possibilitou uma visualização fiel da peça scaneada, possibilitando ressaltar as características de forma e textura. Os modelos virtuais renderizados podem ser visualizados na Figura 6, a escarradeira e Figura 7, o urinol.
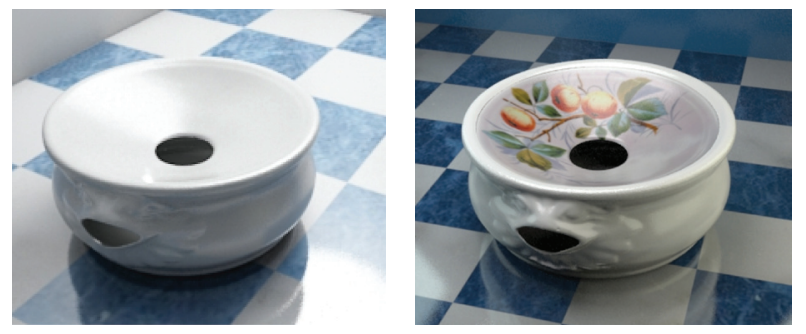

Figura 6. Renderização da escarradeira
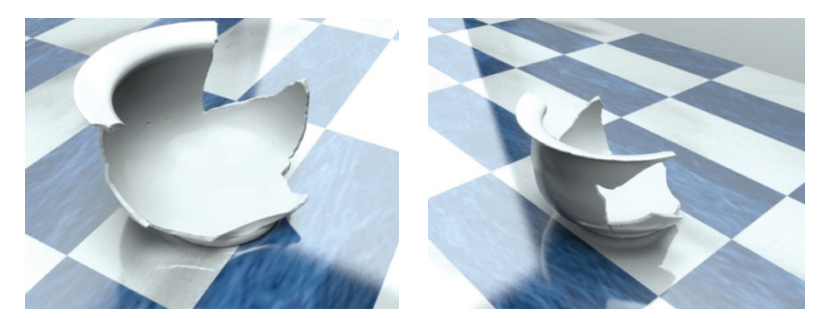

Figura 7. Renderização do urinol 
A renderização das peças acrescentou os detalhes característicos, o que proporcionou a total semelhança com a peça original, conferindo grande qualidade tanto na digitalização e na renderização, garantindo a utilização das peças como modelos virtuais fidedignos.

\section{Conclusões}

Pode-se concluir que a digitalização 3D apresenta-se como uma técnica que vem a contribuir significativamente para que os museus possam, cada vez mais, se aproximar do público, como no caso deste trabalho, disponibilizando as suas peças virtualmente em 3D.

Dessa maneira o presente trabalho contribuiu para a divulgação e conservação de duas peças importantes do acervo do Museu Municipal de Porto Alegre Joaquim Felizardo, a escarradeira e o urinol. Por meio da utilização das técnicas de digitalização tridimensional, os modelos virtuais podem servir de instrumento para restauros reais ou virtuais das peças expostas.

E sendo assim, abre-se caminho para a digitalização de outras peças e para contribuir com a inclusão de museus no ambiente virtual, tornando acessível a qualquer lugar do mundo, via internet.

\section{Referências}

ARBACE, L. et al. Innovative uses of 3D digital technologies to assist the restoration of a fragmented terracotta statue. Journal of Cultural Heritage, Volume 14, Issue 4, July-August 2013, p. 332-345. Disponível em: <http:// www.sciencedirect.com/science/ article/pii/S1296207412001070>.Acesso em: jun. 2013.

BETTS, M. W. et al. Virtual zooarchaeology: building a web-based reference collection of northern vertebrates for archaeofaunal research and education. Journal of Archaeological Science, Volume 38, Issue 4, April 2011, p. 755.e1-755.e9. Disponível em: <http://www.sciencedirect.com/science/article/ pii/S0305440310002153>. Acesso em: jul. 2013.

BORGEAT et al. Visualizing and Analyzing the Mona Lisa. Real-Time Interaction with Complex Models. Published by the IEEE Computer Society, November/December, 2007. 
GODIN, G. et al. Active Optical 3D Imaging for Heritage Applications. Computer Graphics in Art History and Archaeology. p. 24 - 36. September/ October, 2002.

PREFEITURA MUNICIPAL DE PORTO ALEGRE. Museu Joaquim José Felizardo. Disponível em: <http://www2.portoalegre.rs.gov.br/smc/default. php?reg=4\&p_secao=19>. Acesso em: ago. 2013.

SILVA, F. P. da. O uso da digitalização tridimensional a laser no desenvolvimento e caracterização de texturas aplicadas ao design de produtos. 2006. 179 f. Dissertação (Mestrado) - Curso de Pós-graduação em Engenharia Minas, Metalúrgica e de Materiais, Universidade Federal do Rio Grande do Sul, Porto Alegre, 2006. Disponível em: <http://hdl.handle.net/ 10183/10388>. Acesso em: 12 jul. 2013.

SILVA, F. P. da. A Digitalização Tridimensional Móvel e sua Aplicação no Design de Produto. Design \& Tecnologia, Volume 1, Número 01, 2010. Disponível em: <http://www.pgdesign.ufrgs.br/designetecnologia/index. php/det/article/view/7>. Acesso em: jun. 2013.

SILVA, F. P. da. Usinagem de espumas de poliuretano e digitalização tridimensional para fabricação de assentos personalizados para pessoas com deficiência. 2011. 192 f. Tese (Doutorado) - Curso de Pós-graduação em Engenharia Minas, Metalúrgica e de Materiais, Universidade Federal do Rio Grande do Sul, Porto Alegre, 2011. Disponível em: <http://hdl.handle.net/ 10183/36040>. Acesso em: jul. 2013.

TOCCHETTO, F. B. Dados escarradeira e urinol. [mensagem pessoal] Mensagem recebida por: <Wagner Soares Rossi>. em: 12 jun. 2013. 
Recebido em: 10/06/2013

Aceito em: 01/10/2013

\section{Como citar}

ROSSI, Wagner Soares; SILVA, Fábio Pinto da; KINDLEIN JÚNIOR, Wilson. A utilização de modelos 3D para a preservação e divulgação de peças do patrimônio histórico e cultural: estudo de caso com escarradeira e urinol. Arcos Design. Rio de Janeiro: PPD ESDI - UERJ. Volume 7 Número 2 Dezembro 2013. pp. 69-79. Disponível em: [http://www.e-publicacoes.uerj.br/index.php/ arcosdesign]

\section{DOI}

10.12957/arcosdesign.2013.12182

\section{(c) (i) (2)}

A Revista Arcos Design está licenciada sob uma licença Creative Commons Atribuição - Não Comercial - Compartilha Igual 3.0 Não Adaptada. 